\title{
Primary Cardiac Involvement in Scleroderma and Role of Cardiac MRI
}

\author{
Kashif Hussain, Robert C. Stansbury \\ Section of Pulmonary and Critical Care Medicine, West Virginia University School of Medicine, \\ Morgantown, USA \\ Email: khussain@hsc.wvu.edu
}

Received 15 September 2014; revised 12 October 2014; accepted 10 November 2014

Copyright (C) 2014 by authors and Scientific Research Publishing Inc.

This work is licensed under the Creative Commons Attribution International License (CC BY). http://creativecommons.org/licenses/by/4.0/

(c) (i) Open Access

\begin{abstract}
Systemic sclerosis (scleroderma) is a connective tissue disease characterized by vascular dysfunction and fibrosis that can affect multiple organ systems. We present case of primary cardiac involvement and the diagnostic role of cardiac MRI. Cardiovascular magnetic resonance imaging (MRI) is an accurate, quantitative method for the non-invasive assessment of myocardial perfusion. The presence of clinically apparent myocardial involvement in scleroderma portends a very poor prognosis. One study of US veterans found that clinical cardiac disease in scleroderma was associated with a $70 \%$ mortality rate at five years. Management of heart failure and conduction system abnormalities in scleroderma is similar to other cardiac disease. It includes afterload reduction, beta-blockade, defibrillator placement, etc. Patients with reduced cardiac function and normal coronary arteries may benefit from increased immune suppresion.
\end{abstract}

\section{Keywords}

Scleroderma, Primary Cardiac Involvement, Cardiac MRI

\section{Introduction}

Cardiac involvement is typically subclinical in scleroderma. Although cardiac abnormalities could be more prevalent and severe in the diffuse cutaneous subtype of the disease, which has been the most intensively investigated, there is increasing evidence suggesting that cardiac involvement is also a frequent finding in the limited cutaneous subtype.

"Primary" myocardial involvement, without systemic or pulmonary hypertension and without significant renal or pulmonary involvement, may be the consequence of the characteristic vascular lesions of this disease.

Myocardial fibrosis may occur in advanced disease with the fibrotic lesions in a patchy distribution through- 
out both ventricles and not consistent with large coronary artery distribution.

High-resolution perfusion MRI techniques can be used to identify small subendocardial defects. MRI may also allow non-invasive coronary reserve determination and the evaluation of fibrotic myocardium compared with viable tissue [1].

Of note the fibrosis in cardiac scleroderma can also be distinguished from the fibrosis resulting from CAD in that fibrosis secondary to scleroderma can involve the immediate subendocardial layer (typically spared in atherosclerosis) and hemosiderin deposits (commonly observed in atherosclerotic disease) are not appreciated [2].

\section{Case Presentation}

43-year-old female with anti-Scl-70 positive scleroderma was referred to our pulmonary clinic for evaluation of worsening dyspnea.

\subsection{Presentation}

Patient had rapid progression of her disease over one year prior to our evaluation including auto-amputation of digits secondary to Raynaud's, weight loss, esophageal dilatation, and progressive dyspnea. Patient also had significant smoking history as well as asthma previously well controlled on prn SABA. Our initial concern was the development of pulmonary hypertension versus development of interstitial lung disease secondary to scleroderma.

\subsection{Evaluation}

Pulmonary function testing revealed mild restriction with a severe decrease in diffusion capacity. Cardiac ECHO revealed an ejection fraction of 50\% - 55\% and a peak right ventricular systolic pressure of $25-30 \mathrm{~mm} \mathrm{Hg}$. HRCT did not reveal any parenchymal lung disease or other abnormalities. Bronchoscopy with BAL was unremarkable and did not support alveolitis.

\subsection{Interim}

She was evaluated by rheumatologist while in the hospital. Patient had previously been initiated on Prednisone $60 \mathrm{mg}$ daily without significant response. Unfortunately developed acute exacerbation of Raynaud's with skin necrosis and secondary infection preventing the initiation of cytoxan therapy. Two months later she was admitted to hospital with worsening shortness of breath and hypoxia. Echocardiogram showed an ejection fraction of $5 \%-10 \%$.

\subsection{Work-Up}

Given rapid progression of systolic dysfunction patient underwent further cardiac evaluation including cardiac MRI (Figure 1, Figure 2) and myocardial biopsy. MRI revealed an ejection fraction of $8 \%$ with late gadolinium study revealing diffuse RV and LV global enhancement with more prominent inferior and lateral epicardial enhancement. This pattern is not consistent with CAD and is diagnostic of marked cardiac inflammatory pattern involving both ventricles. Myocardial biopsy was unremarkable.

\subsection{Conclusion}

Heart failure treatment was optimized and she was discharged home with life vest. Later she developed syncope secondary to ventricular arrhythmia, leading to placement of ICD. Over next few weeks she experienced multiple ICD shocks secondary to ventricular arrhythmias. Considering her rapid clinical deterioration with poor prognosis she opted for home hospice

\section{Discussion}

- Systemic sclerosis (scleroderma) is a connective tissue disease characterized by vascular dysfunction and fibrosis that can affect multiple organ systems. Cardiac involvement is typically subclinical.

- All cardiac structures may be affected, resulting in pericardial effusion, arrhythmias, valvular impairment, myocardial ischemia, conduction abnormalities, and heart failure [3]. 


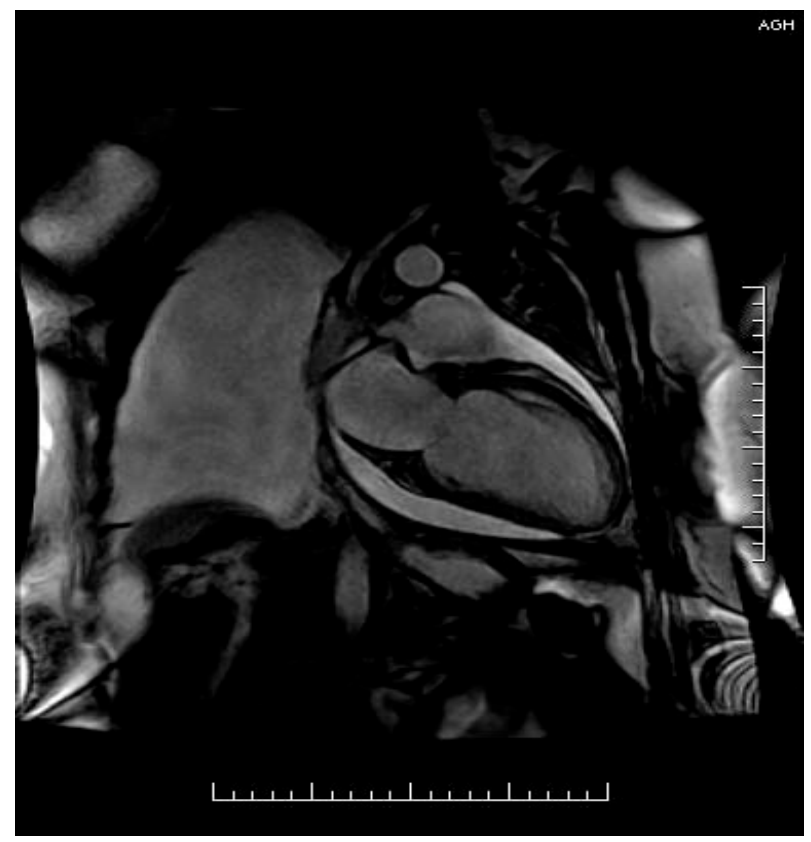

Figure 1. Two chamber view illustrating pericardial effusion.

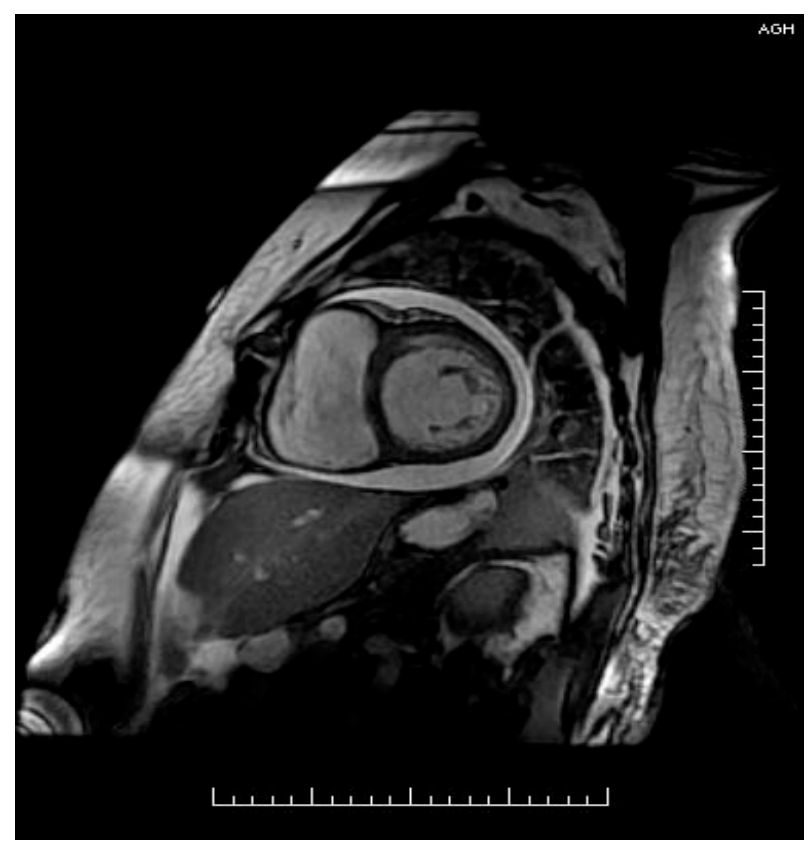

Figure 2. Two chamber view revealing LV and RV remodelling as well as circumferential pericardial effusion. In addition, there is slight evidence of RV pressure (septal D-shape).

- Myocardial fibrosis may occur in advanced disease with the fibrotic lesions in a patchy distribution throughout both ventricles and not consistent with large coronary artery distribution.

- Of note the fibrosis in cardiac scleroderma can also be distinguished from the fibrosis resulting from CAD in that fibrosis secondary to scleroderma can involve the immediate subendocardial layer (typically spared in atherosclerosis) and hemosiderin deposits (commonly observed in atherosclerotic disease) are not appreciated [2].

- Cardiovascular magnetic resonance imaging (MRI) is an accurate, quantitative method for the non-invasive 
assessment of myocardial perfusion. High-resolution perfusion MRI techniques can be used to identify small subendocardial defects. These defects do not correspond to any epicardial coronary artery distribution, and are therefore highly suggestive of microvascular alteration confirming previous hypotheses. MRI may also allow non-invasive coronary reserve determination and the evaluation of fibrotic myocardium compared with viable tissue [1].

- Management of heart failure and conduction system abnormalities in scleroderma is similar to other cardiac disease. This management includes afterload reduction, beta-blockade, defibrillator placement, etc.

- Patients with reduced cardiac function and normal coronary arteries may benefit from increased immunosuppresion.

- The presence of clinically apparent myocardial involvement in scleroderma portends a very poor prognosis. One study of US veterans found that clinical cardiac disease in scleroderma was associated with a $70 \%$ mortality rate at five years [4].

\section{References}

[1] Nagel, E., et al. (2003) Magnetic Resonance Perfusion Measurements for the Noninvasive Detection of Coronary Artery Disease. Circulation, 108, 432-437. http://dx.doi.org/10.1161/01.CIR.0000080915.35024.A9

[2] Kahan, A. and Allanore, Y. (2006) Primary Myocardial Involvement in Systenmic Sclerosis. Rheumatology, 45, iv14iv17. http://dx.doi.org/10.1093/rheumatology/kel312

[3] Champion, H.C. (2008) The Heart in Scleroderma. Rheumatic Diseases Clinics of North America, 34, 181-190. http://dx.doi.org/10.1016/j.rdc.2007.12.002

[4] Medsger, T.J. and Masi, A. (1973) Survival with Scleroderma. II. A Life-Table Analysis of Clinical and Demographic Factors in 358 Male US Veteran Patients. Journal of Chronic Diseases, 26, 647-660. http://dx.doi.org/10.1016/0021-9681(73)90054-4 
Scientific Research Publishing (SCIRP) is one of the largest Open Access journal publishers. It is currently publishing more than 200 open access, online, peer-reviewed journals covering a wide range of academic disciplines. SCIRP serves the worldwide academic communities and contributes to the progress and application of science with its publication.

Other selected journals from SCIRP are listed as below. Submit your manuscript to us via either submit@scirp.org or Online Submission Portal.
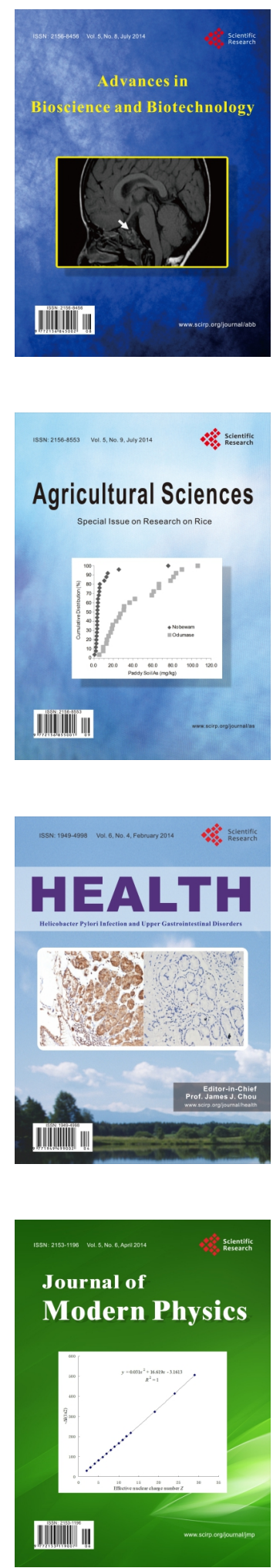
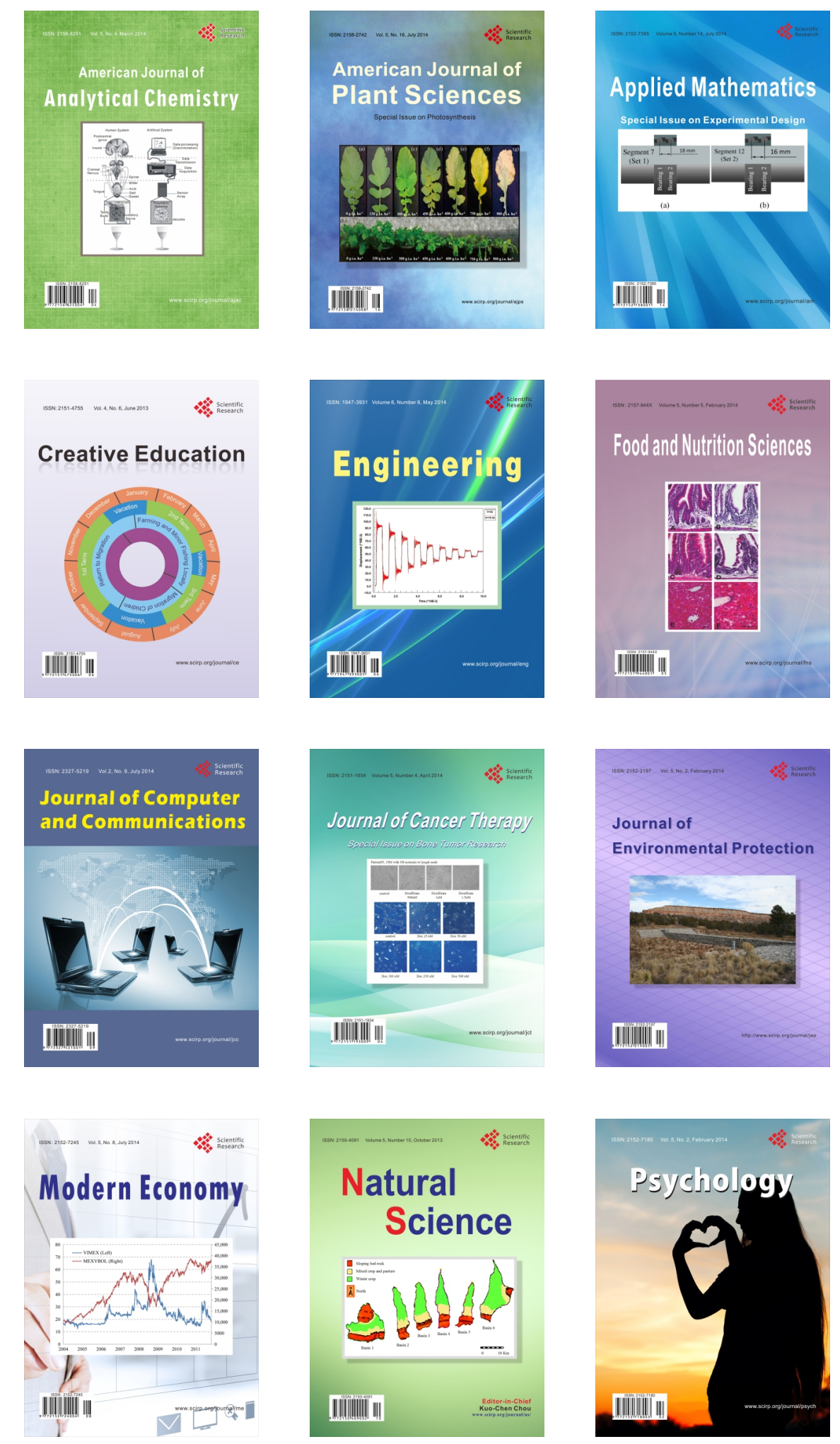